\title{
IMPROVED METHODS FOR EVALUATION OF VISIBILITY FOR INDUSTRIAL VEHICLES TOWARDS SAFETY STANDARDS
}

\author{
Soumitry J. Ray ${ }^{1}$, *Jochen Teizer ${ }^{1}$, Roger Bostelman ${ }^{2}$, Mike Agronin $^{3}$, and Dominic Albanese ${ }^{3}$ \\ ${ }^{1}$ Georgia Institute of Technology \\ 790 Atlantic Dr. N.W. \\ Atlanta, GA 30332-0355, United States \\ (*Corresponding author: teizer@gatech.edu) \\ ${ }^{2}$ National Institute of Standards and Technology \\ 100 Bureau Drive, MS 8230 \\ Gaithersburg, MD, 20899, United States \\ ${ }^{3}$ Direct Dimensions \\ Incorporated, Owings Mills \\ MD, United States
}

\begin{abstract}
Poor visibility of powered industrial vehicles, such as forklifts, used in industry is often the cause of accidents that include pedestrians. Current standards allow up to $20 \%$ non-visible regions for forklifts where measurement of these regions is performed by using lamps. A collaboration of research organizations, including National Institute of Standards and Technology, Georgia Institute of Technology (NIST), and Direct Dimensions, has been evaluating advanced methods for measuring a forklift operator's visibility. These methods can potentially improve visibility standards. They can also help forklift and sensor manufacturers to determine (1) how visibility-assist sensors and algorithms can be designed and (2) where sensors can be mounted on forklifts. This paper includes explanation of visibility measurement experiments performed and results, associated language suggested to standards organizations, and a prototype design for measuring the visibility of forklifts automatically.
\end{abstract}

\section{KEYWORDS}

Blind spots, Construction Workers, Construction Equipment, Vehicle Operator Visibility.

\section{INTRODUCTION}

Eighty percent of forklift accidents involve pedestrians. Such accidents occur on average of once every three days (Mark, 2009). American National Standards Institute/Industrial Truck Standards Development Foundation (ANSI/ITSDF) B56.11.6 "establishes the conditions, procedures, equipment and acceptability criteria for evaluating visibility of powered industrial trucks". The ANSI/ITSDF B56.5 (2012) standard, which references B56.11.6, "defines the safety requirements relating to the elements of design, operation, and maintenance of powered, not mechanically restrained, unmanned automatic guided industrial vehicles and automated functions of manned industrial vehicles.” ANSI/ITSDF B56.11.6 (2005) will soon be harmonized with the International Organization for Standardization/Final Draft International Standard (ISO/FDIS) 13564-1 (2012) and therefore, the ISO standard was also used as guidance for this 
research. The B56.11.6 standard allows up to $20 \%$ of the regions surrounding a forklift to be obscured from the operator. Nonetheless, operators are typically declared at fault for the accidents. NIST supports the development of B56 standards by performing measurements towards improved test methods and serving on the B56.5 standards committee, which includes manned forklifts with automated functions.

The NIST Mobile Autonomous Vehicles for Manufacturing (MAVM) Project has been researching forklift safety (Bostelman, 2009; Bosteman and Liang; Bostelman and Shackleford, 2009) because forklift accident rates are continuing to increase. MAVM is investigating the potential for using onboard sensors integrated with semi-autonomous vehicle control to detect obscured regions and improve safety. However, B56.11.6 measurement methods provide only information about how much of the area is not visible to the operator. Advanced operator visibility measurement technology and methods could potentially map obscured regions, as well as suggest sensor mounting locations and the fields-of-view (FOV) needed to maximize the likelihood of detecting obstacles in those regions.

The current ANSI/ITSDF B56.11.6: 2005 standard, soon to be called B56.11.6.1, and the ISO/FDIS 13564-1 (2012) standard include a test method that uses a row of lights positioned where a forklift operator's head would be and shadows cast by the lights on a test board marked with a grid pattern. The shadows are equivalent to occluded regions and are quantified by counting the grid blocks. Measurements are referenced to the seat index point (SIP) which is located on a standard apparatus fixed to the seat and considered to be equivalent to the intersection on the central vertical plane through the seat centerline of the theoretical pivot axis between a human torso and thighs. Figure 1 shows a test setup on a NIST-owned forklift showing (a) the SIP apparatus and weight bar, (b) the light bar, and resultant shadows from the light bar (c) on a test board as specified in the standard and (d) on a mannequin instead of the test board. That test method shows that there are occluded regions, but does not specify their exact locations. Occlusion is caused by vehicle self-obstructions. Advanced imagers could be mounted to detect obstacles/pedestrians in these occluded regions. The current light method provides only the direction having occluded regions and not the positions on the forklift where sensors might be mounted to compensate for the occlusions.

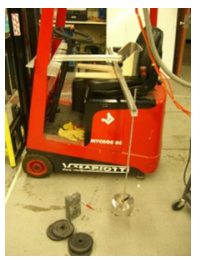

(a)

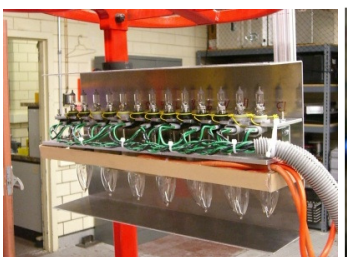

(b)

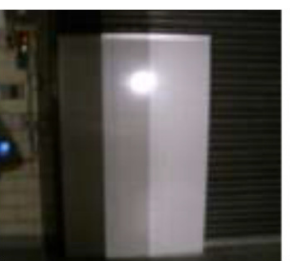

(c)

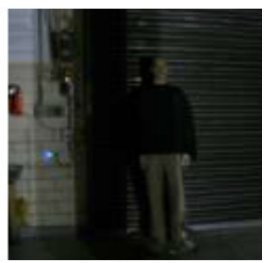

(d)

Figure 1 - Test setup on a NIST-owned forklift showing (a) the seat index point (SIP) apparatus and weight bar, (b) the light bar, and resultant shadows from the light bar (c) on a test board as specified in the standard and (d) on a mannequin instead of the test board.

Exact blind spot causes from vehicle hardware and sensor field-of-view (FOV) can provide vehicle manufacturers with more knowledge about how to design a safe vehicle or to retrofit an existing vehicle with safety sensors. Ideally, an automatic system could be designed to measure vehicle blind spots, allow virtual blind spot display, and can provide this knowledge to the operator. NIST and Direct Dimensions, Incorporated (DDI) have been performing measurements of forklifts to develop an automated visibility measurement system for industrial vehicles using advanced methods" (Agronin and Albanese, 2012). The measurement methods include three approaches: (1) use a computer aided design (CAD) model of an industrial vehicle provided by the original vehicle manufacturer and imported into a 3D rendering tool for analysis, (2) laser-scan the vehicle to create a 3D model equivalent to a CAD model, which can be imported into the 3D rendering tool for analysis, or (3) create a panoramic photo from the driver's eye position, process the image, and import it into the modeling software to make the same visibility measurements per the standard. 
Georgia Institute of Technology (GIT) has also been performing visibility measurements of construction equipment (Teizer et al., 2010a and 2010b, Hinze and Teizer 2011). Blind spots on construction equipment result in poor operator visibility and are one of the leading causes of contact collisions in the construction industry. Six percent of all occupational injuries were from workers being struck by vehicles. Research at GIT has also uncovered new visibility measurement approaches that do not rely on existing equipment CAD models and thus have the potential to solve limitations in reported relevant safety statistics and current standards. GIT's visibility measurements and analysis included: data from laser scanning of the vehicle used as input for an automated blind spot measurement tool, a raytracing algorithm, grid representation of the vehicle, and the development of an automated blind spot measurement tool. The results show the visibility of personnel on the ground from the vehicle operator's perspective. Typically raw laser scan data of equipment yields millions of points depending upon the resolution of the scan (Teizer et al., 2010b). A recent study by Ray and Teizer (2013) focused on reducing computational costs incurred due to the size of the laser scan data. This was achieved by computing the blind spots in a spherical coordinate system instead of a cartesian coordinate system. Owing to the inherent nature of the ray casting algorithm, a significant performance benefit was achieved. The approach was validated on 36 synthetic point clouds for which the ground truth (actual locations) of the blind spots were known a-priori. Furthermore, the developed methodology allows computing different facets of blind spot such as: (a) volumetric blind spot, (b) blind spots map, (c) rectangular 1m boundary visibility, (d) $12 \mathrm{~m}$ circumference analysis, and (e) worker visibility analysis.

The DDI approach is explained in this paper, which includes a detailed workflow for each of the three approaches, results from measuring a NIST forklift, and block diagrams showing how to automate the visibility measurement analysis using modeling and rendering. Also included in this paper is an explanation of the new GIT analysis approach and results using the DDI collected data of the NIST forklift. A comparison of the DDI and GIT approaches follows along with suggested standard test method language for potential proposal to the ANSI/ITSDF B56.11.6.1 standard committee.

\section{EXPERIMENTS}

Operator visibility measurements were performed using a NIST-owned forklift having a $4000 \mathrm{~kg}$ capacity and $5 \mathrm{~m}$ lift height. Four different approaches were tested and are explained in this section: (1) Using a computer aided design (CAD) model of an industrial vehicle provided by the original vehicle manufacturer and imported into a 3D rendering tool for analysis, (2) Laser-scanning the vehicle to create a 3D model equivalent to a CAD model, which can be imported into the 3D rendering tool for analysis, (3) Importing data created from a panoramic photo taken from the driver's eye position into the modeling software to make the same visibility measurements per the standard, and (4) Using a computational approach on laser scan data of equipment (Ray and Teizer, 2013). All tests were to provide outputs similar to the criteria shown in the ISO/FDIS 13564-1 standard for ride-on forklifts.

\section{Visibility Measurement via CAD Model}

A CAD model can be used directly to measure visibility rather than to measure the actual vehicle. All subsequent measurement approaches are based on this, unless stated otherwise. The CAD model must be a solid model that includes any surface visible from the cabin, plus the seat, mast, and fork tines. The $\mathrm{CAD}$ model must be configured with the boom and forks in the proper position for measurements to meet the required standards. The current ANSI standard requires the mast to be angled back and the forks to be within a certain height range. The ISO standard, however, requires two mast positions: vertical and angled back. The vehicle and SIP dimension measurements are then required to determine the location of the lamps and the projection screens to meet the required standards. Length and width of vehicle can be obtained directly from the CAD model. The SIP dimensions may be known to the manufacturer or may need to be measured using a fixture on an actual vehicle. The SIP should be determined relative to a reference point in the CAD model, such as the floor, left extents of the vehicle, and front face of the vehicle. Then the CAD model is saved to a mesh (polygonal) format which is required for importing the model into an image rendering software ${ }^{1}$. The image rendering software created a $3 \mathrm{D}$ model of each 
projection screen and each lamp in the lamp array. The positions of screen and each lamp, and the number of lamps required, are completely different depending on which visibility standard is used for assessment.

Image rendering software contains a ray-tracing module that can shine virtual light sources through a scene and project the shadows onto a surface. This capability allows users to create a table of lamp positions, orientations, and projection screens that correspond to each of the tests in the standard. For each lighting configuration, shadows are rendered onto the screen and the screen images are saved. The lamps can be assigned a fixed brightness, so that by measuring the gray-scale of the projected screen, one can determine exactly how many lamps in the array are illuminating a particular spot. Only spots with zero brightness are in full shadow from all the lamps. This makes the technique superior to using actual lamps, where the edge of a shadow may be ambiguous. Figure 2a shows a screen shot of the virtual lamps (black and yellow triangles) shining through the forklift CAD model onto a virtual screen. Therefore, the virtual lamps that are blocked by vehicle structure can provide additional non-visible viewpoint information than what is currently requested by the standard. The final step in the measurement process was to analyze each rendered screen as per the standard. For most of the tests in the ANSI standard, a $500 \mathrm{~mm} \times 500 \mathrm{~mm}$ box is moved through every possible position on the screen. The vehicle fails the test if more than $80 \%$ of the box area is in shadow. The ISO standard required less than $80 \%$ shadow in a $500 \mathrm{~mm} \times 1200 \mathrm{~mm}$ area. This step can be automated with a programming language that includes a powerful image analysis library according to the standard's requirements. Determining the percentage of black pixels (black pixels denote shadows) within a specified area in an image is relatively easy to implement. Figure $2 b$ shows the sample image of ANSI/ITSDF B56.11 test 2a, straight ahead and down, with the area with maximum shadow outlined in red (black pixels denote shadows).

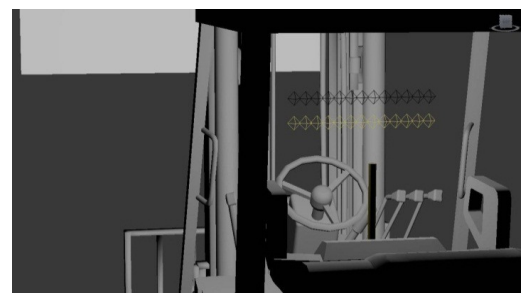

(a)

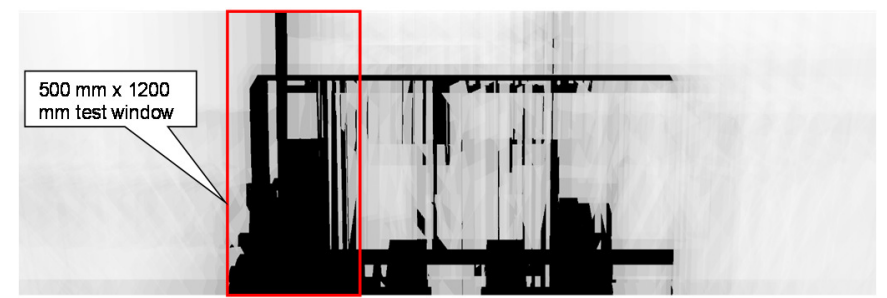

(b)

Figure 2 - (a) Screen shot of the virtual lamps (black and yellow triangles) shining through the forklift CAD model onto a virtual screen. (b) Sample image showing area with maximum shadow.

\section{Visibility Measurement via Meshed Model of Laser Scan}

Laser scanning can capture the geometry of an object with millimeter accuracy and resolution. This is an ideal tool for creating a 3D model of a complex object. Then a fixture built by NIST to locate the SIP was utilized. The fixture was loaded with a spring scale (lateral load) and weights as per ISO 5353. The sample vehicle was scanned with a spherical laser scanner. Spheres were attached to the vehicle and the surrounding floor to assist with the alignment of scans taken from different points of view. The scanner was positioned at eleven locations around the outside of the forklift to capture every surface - inside and outside the cabin of the forklift. Additional scans of the forklift were taken with the SIP fixture installed and with the mast in different positions. To make a complete model from the scans, it is necessary to align or register the overlapping portions of the individual scans. This was performed by using Scene visualizing software to find a mathematically best fit between the overlapping geometry, or by aligning common reference points, such as the spheres attached to the vehicle. The result was a single point-cloud containing the points from all 11 scans. Although some research has been done in this area (Franaszek et al., 2009), this registration process is not fully automatable by current off-the-shelf software, but it is not a difficult process to automate. A mesh model was created by constructing a series of connected triangles from the point cloud. Mesh processing software was used to delete extraneous points and then create a mesh model. CAD objects were fitted to the points, resulting in a to-scale CAD model of the forklift. The additional scan data was used to determine the reference surfaces of the SIP fixture and the mast's axis of 
rotation. CAD tools were then used to construct the SIP, the locations of the lamps, and the projection screens for visibility measurement. A CAD software package was used to measure the length and width of the forklift while creating the mesh model. To determine the SIP coordinates, the CAD software package was used to construct the planes that were fit to the SIP fixture scan data. The SIP was located at the intersection of these planes. The remaining steps for measuring visibility are the same as for measuring visibility with a CAD model (as described in the previous section).

\section{Visibility Measurement via Photo Panograph}

A novel approach for measuring visibility is to use photo panographs from inside the vehicle. A panograph is a panoramic view created by stitching together a series of overlapping individual images. This is the same technique used to assess pilot visibility in aircraft cockpits. A photo panograph can capture all the geometry seen by the driver from within the vehicle. The panographs do not directly measure the depth. However, the azimuth and elevation angles can be determined with considerable accuracy in the panograph. A spherical projection from each panograph is used to mask each virtual lamp, creating the same shadows as the 3D model itself. The SIP fixture was then installed in the same manner as described in the previous Section followed by the installation of the panograph assembly. The assembly consists of a standard Red, Green, Blue (RGB) camera and a programmable motorized camera mount. The motorized camera mount was clamped to a beam at multiple positions to match the position of each lamp in the upper row of the lamp assembly. While this arrangement was sufficient for the forward-facing lamp orientation, another fixture was developed to locate the camera at positions matching the lamp array when it is aimed to the sides or behind the vehicle. Dimensions are taken from the ANSI visibility standard. To recreate the ANSI test standard, it is necessary to position the panograph at each lamp location in the lamp array (26 positions), with the array in every required configuration (seven angles), for a total of 182 panographs. Since the objective was to demonstrate the process and not take complete data, panographs were taken corresponding to only one row of lamps in the forward-aiming orientation. However, seat bolsters obstructed the camera assembly for the outer-most lamp positions. Thus only eleven panographs were taken, along with one additional panograph, at the center position, with the mast tilted back and the forks raised $1.1 \mathrm{~m}$, to create a worst-case obstruction for the driver. The laser-scan data of the panograph setup was used to determine the exact camera position relative to the SIP. The motorized camera mount was programmed to take 16 images around $360^{\circ}$ of azimuth, at two different elevation angles. Successive images need to overlap by about $30 \%$ in order to stitch well. The first step in processing the photos was to stitch all the photographs together into a continuous panorama. After stitching, vertical pixel position maps to an elevation angle, and horizontal pixel count maps to an azimuth angle in the spherical projection. The only manual step in the process is to indicate $0^{\circ}$ azimuth. The support beam was taken as $\pm 90^{\circ}$ azimuth reference, and measured to $0^{\circ}$. The next step was to convert each stitched panograph into masks for the 3D analysis software. By manually tracing the contours and filling it in with black to convert the image in to a black and white image. Figure 3a shows a spherical projection of a panographic view from the driver's eye position and Figure 3b shows a black and white mask of the same panographic image, centered and expanded to $360^{\circ}$ azimuth by $180^{\circ}$ elevation.

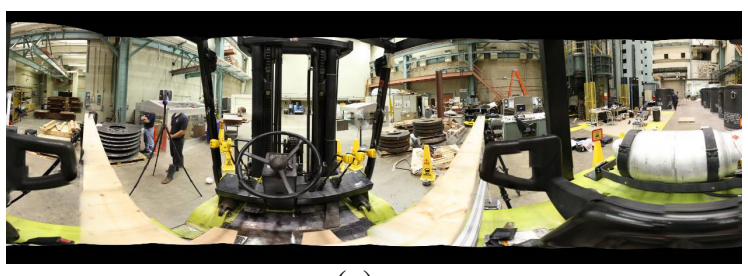

(a)

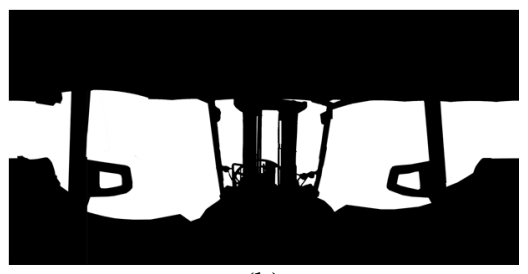

(b)

Figure 3 - (a) Spherical projection of a panographic view from the driver's eye position, (b) Black and white mask of the same panographic image, centered and expanded to $360^{\circ}$ azimuth by $180^{\circ}$ elevation.

The pixel coordinates correspond exactly to the vector from the eye position in polar coordinates.

The pixel coordinates correspond exactly to the vector from the eye position in polar coordinates. The vehicle dimensions are loaded followed in CAD model based visibility measurement. To shade the 
lamps, the panographs are imported as a mask that surrounds each virtual lamp. The mask shades the light exactly as if an actual vehicle were in the way. The image analysis can be performed using the method described in CAD model based approach.

\section{Visibility Measurement via Volumetric Model of Laser Scan (Ray and Teizer, 2013)}

The data input was a laser scan of the forklift. The scan consisted of roughly $20 \times 10^{6}$ points. The point cloud was binned (meaning: represented by a histogram or in simpler terms, a series of buckets) into a three dimensional grid in a spherical coordinate system in steps of size $\Delta r=0.05 \mathrm{~m}, \Delta \theta=0.3^{\circ}$, and $\Delta \phi$ $=0.3^{\circ}$. The numbers of bins along the three directions were: 416 along $r, 1200$ along $\phi$, and 600 along $\theta$. The number of bins is computed from the step-size values input by the user. Due to memory constraints for storing the three dimensional grid, the step sizes were set to the above minimal possible values.

\section{$\underline{\text { Volumetric Blind Spot }}$}

Percentage volumetric blind spot in this research is defined as the ratio of total blind area on the surface of a $12 \mathrm{~m}$ radius sphere to the total area of the same sphere lying above the ground plane. The sphere is assumed to be centered at the origin or the head of the operator. The surface of the sphere lying above the ground plane is only considered during the computation, the volumetric blind spots are illustrated graphically in Figures 4. Both figures show the visible and blind areas on a $12 \mathrm{~m}$ sphere centered at the origin. The percentage volumetric blind spot was $19.48 \%$. The time taken for computing the volumetric blind spot was $1.19 \mathrm{~s}$. The visible areas are shown in green; the blind areas are shown in red.
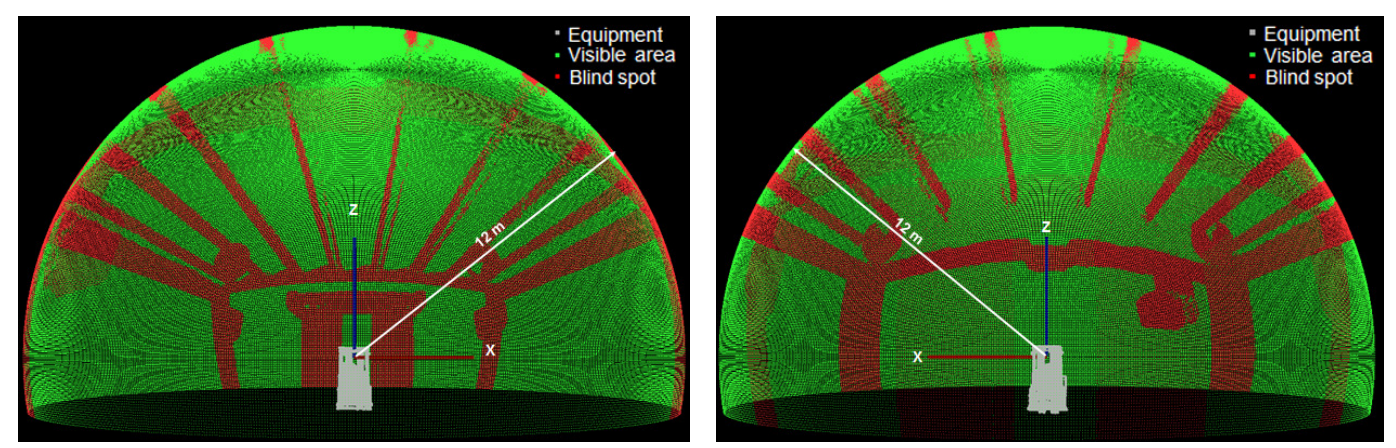

The blind spots a forklift operator experiences looking to the front (left image) and rear (right image)

Figure 4 - Volumetric blind spot on 12m radius sphere. The red areas represent blind spot regions and the visible areas are represented by green color.

\section{$\underline{\text { Blind spots Map }}$}

A blind spots map is the mapping of visible and blind areas contained in a $12 \mathrm{~m}$ radius circle lying on the ground plane, with the operator position at the center. The percentage blind spot area was computed to be $21.20 \%$ (see Figure 5a). The time taken for computation was measured to be $0.83 \mathrm{~s}$ (includes computation of blind spots map, $12 \mathrm{~m}$ circumference visibility, and rectangular $1 \mathrm{~m}$ boundary analysis).

\section{$\underline{12 \mathrm{~m} \text { Circumference Visibility }}$}

The $12 \mathrm{~m}$ circumference visibility measurement is similar to blind spot map measurement as discussed above; however, here visibility is measured only along the edge of the circle and all measurements are in terms of length. The total length of the circumference $(2 \pi r, r=12.0 \mathrm{~m})$ was computed to be $75.40 \mathrm{~m}$ and the visible length along the circumference was $62.71 \mathrm{~m}(83.17 \%)$. Additionally, the software reports the arcs along the circumference that are invisible. Figure $6 \mathrm{a}$ is an annotated graphical representation of the invisible arcs. 


\section{$\underline{\text { Rectangular 1m Boundary Visibility }}$}

Visibility was measured on the circumference of a rectangular $1 \mathrm{~m}$ boundary around the machine. A rectangular $1 \mathrm{~m}$ boundary is constructed at an offset distance of $1 \mathrm{~m}$ from the smallest rectangle that can be placed around the vertical projection of the machine on the test floor (ground level) on which the machine is located. The visible length was computed to be $9.66 \mathrm{~m}$ which constituted $46.78 \%$ of the length of the rectangular $1 \mathrm{~m}$ boundary. Figure $5 \mathrm{~b}$ is a graphical illustration of the visibility along the rectangular $1 \mathrm{~m}$ boundary.

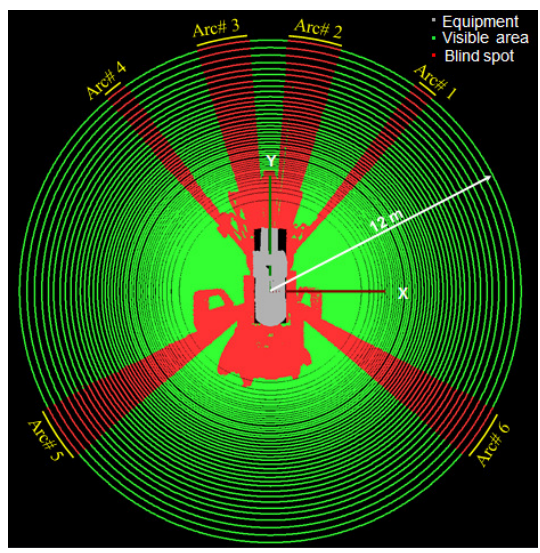

(a) $12 \mathrm{~m}$ Circumference visibility

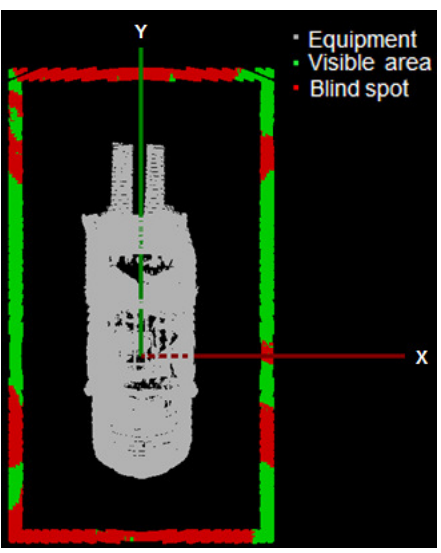

(b) Visibility at rectangular $1 \mathrm{~m}$ boundary

Figure 5 - Plan views of forklift operator's field-of-view.

\section{RESULTS}

The meshed model from the laser scan produces nearly the same shadow projection as the CAD model, as long as care is taken in processing the meshed model to ensure that no actual obstructions are deleted. Figures $6 \mathrm{a}$ and $6 \mathrm{~b}$ show screen projections made with the CAD model and mesh model, respectively. The panograph approach can generate results matching the CAD model approach if all conditions are the same. As discussed earlier, the panographs were taken from a position slightly below that required by the standard. However, in the images presented here, the same lighting positions and number of lights for the CAD model and the panographs are recreated. These images are created with one row of 11 lamps. Figures $6 \mathrm{c}$ and $6 \mathrm{~d}$ show screen projections made with 11 lamps positioned inside the CAD model and inside the panograph, respectively.

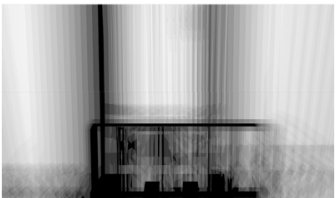

(a)

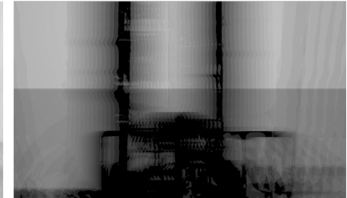

(b)

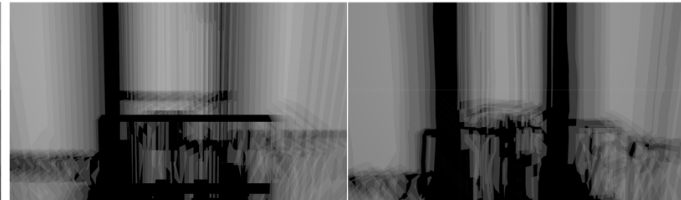

(d)

Figure 6 - Screen projections from (a) CAD model (b) Meshed model (c) Lamps positioned in CAD model and (d) 11 Panographs

\section{CONCLUSIONS}

Results from the visibility measurement experiments demonstrated that if vehicle measurement is required, scanning the vehicle using a 3D laser and producing a CAD model provided the clearest comparison of visible and non-visible regions. It also provided the easiest method tested towards design of an automated visibility measurement system. Laser scan measurements based on approach (Ray and 
Teizer, 2013) provides objective results and visualization of different facets' blind spots. The computations are performed in discretized three-dimensional space. Thus, sub-millimeter accuracy in input data may not be necessary. Other less expensive alternatives might focus in the future on using stereo cameras or range sensors. Latter sensors may be used to develop coarse point clouds as they typically have lower resolution and range compared to commercially-available laser scanners. Feasibility studies and experimental verifications are thus required if researchers or developers proceed in this direction. Presently, laser scanning system cost is higher than the panoramic camera method, which also demonstrated good results.

\section{REFERENCES}

Agronin, M. and Albanese, D. (2012). Visibility Measurement of a Forklift, Solicitation Number SB134112-RQ-0222. NIST Report.

Austin, M. (2009). Fork Lift Awareness. In Performance Metrics for Intelligent Systems Workshop (PerMIS ’09), NIST Special Session.

ANSI/ITSDF B56.5 (2012). Safety Standard for Driverless, Automatic Guided Industrial Vehicles and Automated Functions of Manned Industrial Vehicles.

ANSI/ITSDF B56.11.6 (2005). Evaluation of Visibility from Powered Industrial Trucks

ISO/FDIS 13564-1 -2012 (2012) Powered industrial trucks - Test methods for verification of visibility Part 1: Sit-on and stand-on operator trucks up to and including 10t capacity (Final Draft International Standard).

Bostelman, R. (2009). White Paper: Towards Improved Forklift Safety. Proceedings of the 2009 Performance Metrics for Intelligent Systems Workshop (PerMIS '09), NIST Special Publication 1112.

Bostelman, R., Liang, L.P.. Measurement and Evaluation of Visibility Experiments for Powered Industrial Vehicles. NIST Internal Report \#7837, 201.

Bostelman, R. and Shackleford, W. (2009). Performance Measurements Towards Improved Manufacturing Vehicle Safety. Proceedings of the 2009 Performance Metrics for Intelligent Systems Workshop (PerMIS ’09), NIST Special Pub. 1112.

Direct Dimensions, Inc. (2012.) Cockpit Simulation. Retrieved from Direct Dimensions Incorporated website: http://www.directdimensions.com/port_projects.htm.

Franaszek, C., Cheok, G., and Witzgall, C. (2009). Fast automatic registration of range image from 3D imaging systems using sphere targets. Automation in Construction 18, pp 265-274.

Hinze, J.W. and Teizer, J. (2011). Visibility-Related Fatalities Related to Construction Equipment, Journal of Safety Science, Elsevier, 49(5), 709-718.

Ray, S.J., Teizer, J. (2013). Computing Blind Spots of Construction Equipment: Implementation and Evaluation of an Automated Measurement and Visualization Method. (in review)

Teizer, J., Allread, B.S., Fullerton, C.E., and Hinze, J. (2010). Autonomous pro-active real-time construction worker and equipment operator proximity safety alert system, Automation in Construction, Elsevier, 19, 630-640.

Teizer, J., Allread, B.S., and Mantripragada, U., (2010). Automating the blind spot measurement of construction equipment. Automation in Construction Journal, Elsevier, 19(4), 491-501. 